\title{
Reduced pain during baroreceptor stimulation in patients with symptomatic and silent myocardial ischaemia
}

\author{
Attila Kardos, Harald Rau, Mark W Greenlee, Conrad Droste, Stuart Brody, and \\ Helmut Roskamm
}

\begin{abstract}
Objective: Baroreceptor activation has been shown to reduce pain, and the accumulation of such pain reduction has been implicated in the operant learning (under certain circumstances) of hypertension. The current study is an examination of differences in the pain dampening effects of baroreceptor activity in patients with symptomatic and asymptomatic myocardial ischaemia. The objective was to determine whether there are differences between patients with symptomatic and silent myocardial ischaemia with respect to their antinociceptive response to baroreceptor stimulation, and, if so, whether these differences could be related to the absence of angina pectoris pain in patients with silent myocardial ischaemia. Methods: Sensory detection and electrical pain thresholds were compared in nine symptomatic and 10 asymptomatic patients with replicable myocardial ischaemia during PRES (phase related external suction) carotid baroreceptor manipulation in which the pressure inside a neck cuff was phase locked in time to the R wave of the ECG and negative pressure was applied during either systole or diastole. Tourniquet pain thresholds were also determined. Results: It was found that (1) external baroreceptor manipulation had no effect on detection thresholds; (2) painful stimuli were judged by both symptomatic and asymptomatic patients as less intense when delivered during maximum baroreceptor activity; (3) symptomatic and asymptomatic patients did not differ in their sensory detection thresholds; and (4) asymptomatic patients had significantly higher ischaemic (tourniquet) pain thresholds than symptomatic patients. Conclusions: The results indicate that baroreceptor activity can modify the intensity of painful stimuli. The degree to which baroreceptor manipulation affects pain does not appear to differ between patients with painful and silent myocardial ischaemia. Thus the baroreceptor dependent pain inhibition effects seems not to be responsible for the higher ischaemic pain threshold found in the silent myocardial ischaemia group.

Cardiovascular Research 1994;28:515-518
\end{abstract}

A ngina pectoris pain often accompanies an acute myocardial ischaemic event, and as such serves as an important warning to discontinue strenuous physical activity. ${ }^{1}$ However, some patients do not experience angina pectoris pain during acute myocardial ischaemic attacks. This phenomenon is referred to as silent myocardial ischaemia. Approximately $1-3 \%$ of patients with coronary heart disease have never experienced angina pectoris pain despite the presence of objective signs of pronounced, transient myocardial ischaemia. ${ }^{1-3}$ In about $30 \%$ of patients with coronary heart disease, marked myocardial ischaemic events, as evidenced by changes in the ST segment of the electrocardiogram, occur without concomitant pain. ${ }^{4}$

Differences in pain sensitivity between patients and painful and painless myocardial ischaemia have been reported for ischaemic, ${ }^{34}$ and electrical pain tests. ${ }^{56}$ Personality inventories describe asymptomatic patients as less nervous, less excitable, and more masculine that symptomatic patients. ${ }^{3}$ It remains unclear to what extent hormonal and/or neural factors underlie the absence of pain in silent myocardial ischaemia.

There is a growing literature on the relationship of the cardiovascular system to pain. The carotid baroreceptors play a role in both cardiovascular homeostasis and pain regulation. ${ }^{7}$ In the current study we investigated whether there may be differences in the antinociceptive activity of the baroreceptors in patients with symptomatic and asymptomatic myocardial ischaemia.

Coronary heart disease is not the only cardiovascular disease that has been associated with altered pain perception. There are numerous reports of hypertension having such an association. ${ }^{8-11}$ The modulatory effect of baroreceptor activity on pain sensitivity may also be altered in borderline hypertension. $^{12}$ It has been proposed ${ }^{8}$ that the negatively reinforcement (by baroreceptor activation dependent pain and stress reduction) afforded by repeated transient increases in blood pressure leads to the operant learning of hypertension. This learning may first manifest as increased cardiovascular reactivity.

The aim of the present study was to determine whether there are differences in patients with symptomatic and silent myocardial ischaemia with respect to the antinociceptive effects of baroreceptor activation. The study also examined the following questions. Is there a difference in pain detection threshold between silent and non-silent ischaemic patients? Does external baroreceptor manipulation affect detection thresholds in patients with silent and non-silent myocardial ischaemia? Is there a difference between silent and non-silent ischaemic patients in tourniquet ischaemic pain thresholds?

Institute for Medical Psychology and Neurobiology, University of Tübingen, Gartenstrasse 29, D 72074 Tübingen, Germany: S Brody, H Rau; Benedikt-Kreutze Herz-Kreislauf-Rehabilitationszentrum, Bad Krozingen, Germany: A Kardos, M W Greenlee, C Droste, H Roskamm. Correspondence to Dr Rau. 
Table 1 Clinical details of the patients studied.

\begin{tabular}{|c|c|c|c|}
\hline & $\begin{array}{l}\text { Asymptomatic } \\
(n=I 0)\end{array}$ & $\begin{array}{l}\text { Sumptomatic } \\
(n=9)\end{array}$ & $\begin{array}{l}\text { Total } \\
(n=19)\end{array}$ \\
\hline $\begin{array}{l}\text { Transient nyocardial ischaemic } \\
\text { events }^{2} \text { (without AMI) }\end{array}$ & 6 & 4 & 10 \\
\hline with anterior $\mathrm{AMI}^{\circ}$ & 1 & + & 5 \\
\hline with posterior $\mathrm{AMI}^{\mathrm{a}}$ & 3 & 1 & 4 \\
\hline Coronary spasms without stenosis & 2 & 0 & 2 \\
\hline One vessel disease & 4 & 3 & 7 \\
\hline Two vessel disease ${ }^{2}$ & 0 & 3 & 3 \\
\hline Three vessel disease & 4 & 3 & 7 \\
\hline Age (years) & 55.5 & 55.4 & 55.5 \\
\hline $\mathrm{BP}$ diastolic ( $\mathrm{mm} \mathrm{Hg}$ ) & 82.5 & 81.7 & 82.1 \\
\hline BP systolic (mm Hg) & 123.0 & 124.4 & 123.7 \\
\hline
\end{tabular}

Number of patients ever having such events.

$\mathrm{AMI}=$ acute myocardial infarction: $\mathrm{BP}=$ blood pressure.

\section{Methods}

Subjects

A group of nine patients with symptomatic myocardial ischaemia and 10 patients with asymptomatic myocardial ischaemia participated in the experiment. Coronary heart disease was verified in all patients by angiographic examination. Severity of coronary stenosis was $50 \%$ or more in at least one of the major coronary vessels (table I). The patients were drawn from a large patient population in the Cardiac Rehabilitation Centre in Bad Krozingen. Exclusion criteria were neurological. psychiatric, or endocrine (for example diabetes) disorders. All patients had been taking standard medication (nitrates, calcium antagonists, $\beta$ blockers). Medication was discontinued 24 to $48 \mathrm{~h}$ prior to the investigation. Classification of the subjects into the symptomatic or asymptomatic groups was based on reports about daily chest pain and additionally, on the pain reports given during exercise tests. Patients who repeatedly showed objective signs of ischaemic events during exercise tests (significant ST segment depression in the electrocardiogram and/or a significant increase in diastolic pressure within the pulmonary artery) without concomitant pain were classified as asymptomatic patients. Patients were not aware of either the hypotheses being tested or their group assignment. A description of the subjects' ages, blood pressure values, and severity of coronary stenosis is given in table I. The symptomatic and asymptomatic groups did not differ with respect to age or resting blood pressure (all $F$ values $<0.2$ )

The study was approved by the University of Tübingen ethics committee, and subjects were given a written explanation of possible hazards and signed an informed consent form. The investigation conforms with the principles outlined in the Declaration of Helsinki.

\section{PRES baroreceptor manipulation}

During systole the highest level of endogenous baroreceptor activity occurs, while such activity is low during diastole. These natural fluctuations can be augmented by PRES (phase related external suction), which increases firing of the baroreceptors through suction. and decreases such firing by blowing. Superimposition of PRES suction on systole maximises vascular dilatation and thus results in the highest levels of baroreceptor activity, while the application of positive pressure during diastole decreases blood vessel diameter and results in the lowest rate of baroreceptor firing. Unlike earlier neck cuff methods, PRES provides for a control condition as subjects cannot reliably differentiate between conditions."

In the PRES method, ${ }^{\text {it }}$ a malleable neck cuff positioned on the subject provides rapidly alternating air pressure changes of $-30 \mathrm{~mm} \mathrm{Hg}$ to $+10 \mathrm{~mm} \mathrm{Hg}$ as implemented by a computer controlled valve and ai pump system to induce phasic changes in baroreceptor activity (negative cuff pressure sucks the cuff onto the skin. and thus automatically tightens gaps between cuff and tissues; excess pressure increases these gaps and thus increases air leakage, making high positive pressures technically more difficult).

Pressure pulses began $100 \mathrm{~ms}$ after detection of the cardiac $\mathrm{R}$ wave, and were equal in duration to one half of the previously recorded mean interbeat interval minus $100 \mathrm{~ms}$. The diastolic pulse followed the systolic pulse, and was of equal duration. The sequence was repeated for $6 \mathrm{~s}$ in each of the 64 trials.

Two baroreceptor manipulation conditions (resulting in different baroreflex intensities) in conjunction with two different cardiac phase onsets of the electrical stimulus during the systole or diastole were conducted: systolic suction - diastolic blowing, electric stimulus delivered either during the systolic (1) or diastolic phase (2); and systolic blowing - diastolic suction, the stimulus delivered either during the systolic $(3)$ or diastolic phase (4). In the case of systolic electrical stimulus delivery. the stimulus (50 ms duration) was applied $200 \mathrm{~ms}$ after detection of the $\mathrm{R}$ wave of the electrocardiogram. If applied during diastole, stimulus onser was $100 \mathrm{~ms}$ after cuff pressure reversal. The conditions were presened in a pseudorandomised order. Respiration was not controlled
Electrical pain stimuli and psychometric procedures Sensory detection thresholds (the stimulus intensity level at which subjects reliably report any sensation) and the pain ratings (subjective quantification of the intensity of a stimulus felt to be painful) were determined in each of four experimental conditions, the order of which was randomly determined by the computer. There was a total of 64 trials, 16 in each condition. A conventional, two interval psychometric procedure based on an adaptive algorithm (the so called Best PEST [Parametric Estimation by Sequential Testing] method ${ }^{15}$ ) was used for threshold determination. In the Best PEST paradigm, each trial is divided into two time intervals of $3 \mathrm{~s}$ each, which were announced by a clearly audible computer generated tone. The computer algorithm selected on a random basis whether the stimulus was delivered in the first or second interval. A staircase procedure was employed to determine the stimulus amplitude at which the subjects responded correctly in $75 \%$ of the trials. The stimulus amplitude varied from $0.5 \mathrm{~mA}$ to a value well below detection theshold $(0.02 \mathrm{~mA})$ in 40 equal intensity steps. The first trial began with the highest value possible and for each subsequent trial the stimulus amplitude which had the greatest likelihood of corresponding to the actual threshold value was chosen. Detection thresholds were measured in each of the four experimental conditions, the order of which was randomly determined by the computer.

Pain sensitivity was measured by presenting stimuli with an intensity five times greater than the threshold detection level during each of the four baroreceptor manipulation conditions. Subjects rated the intensity of the stimulus after each trial by adjusting a visual analogue scale (moving a potentiometer which had markings for no sensation and pain threshold).

The experiment was conducted in two parts, each on a separate day. In most cases these were consecutive days, in the remaining cases with one day in between. The experiments were performed at the same time of day for each patient. Patients were asked to forego all medication $48 \mathrm{~h}$ prior to investigation, although they were allowed to use nitrates (which have short lived effects). The patients were also instructed to refrain from alcoholic beverages for $24 \mathrm{~h}$ before the study, as well as from caffeinated beverages and tobacco on the day of investigation. All subjects had threshold detection on the first day, and pain ratings on the second experimental day.

\section{Physiological recordings}

The electrocardiogram was measured using Beckman $\mathrm{Ag} / \mathrm{AgCl}$ electrodes which were attached to the thorax at positions producing clear $R$ waves. The $R$ wave of the electrocardiogram was detected by a cardiotachometer and the resulting signal was transmitted to a digital input channel of an input-output board (Data Translation 2821) in an IBM-AT compatible computer. The computer measured the interval between successive $R$ waves with a resolution of $0.5 \mathrm{~ms}$. The interbeat intervals were converted off line to heart rate. The information about timing of $\mathrm{R}$ waves was used for the presentation of both electrical stimuli and cuff pressure changes, as well as the calculation of heart rate responses to the neck cuff manipulation. Tonic stimulation of the baroreceptors (systolic negative, diastolic positive cuff pressure) should elicit the baroreceptor reflex consisting of both heart rate deceleration and blood pressure decrease. ${ }^{16}$ In the present study, heart rate responses were used to verify baroreceptor activity changes.

\section{Experimental procedure}

Patients sat upright in a comfortable examination chair. The electrocardiogram electrodes were positioned on the patient's thorax. For applying the electrical stimuli used during the detection threshold and pain determination procedures, a stainless steel, intracutaneous fingertip electrode was attached after removing a small area of the epidermis with a dental bore. ${ }^{17}$ The neck cuff was next fastened to the patient's neck and secured so as to provide a tight fit without causing any noticeable discomfort. A few practice trials including application of the electrical stimuli and suction and pressure pulses to the neck cuff were run to ensure that the electrodes were properly positioned and that the neck suction and fingertip stimulation techniques were functioning. The patient was familiarised with the action of the neck cuff, and the sounds produced by the computer to cue the onset of a trial. Questions specific to the investigation were responded to by the experimenter; otherwise talking was not encouraged. The experiments were conducted in a quiet room, in which only the experimenter and the patient were present.

\section{Ischaenic toumiquet pain test}

To determine the patients' sensitivity to ischaemia, the tourniquet pain test was performed (the diagnostic procedures are described in ${ }^{18-20}$ ) prior to the threshold determination task on the first day. This test produces ischaemic pain and is similar in this regard to angina pectoris pain. The brachial artery was occluded by inflating a Riva Rocci cuff bound around the upper arm to $220 \mathrm{~mm} \mathrm{Hg}$. A hand grip task was performed at a rate of 60 contractions $\cdot \mathrm{min}^{-1}$. The time elapsing until pain was reported by the patients was measured as an indication of pain threshold. 
Data analysis

Data acquisition, control of experimental stimuli, and baroreceptor manipulation were under the control of a IBM-AT compatible computer with a computer program written in ASYST (Keithley-Metrabyte, Rochester, New York). Heart rate responses and pain ratings were averaged separately for the four experimental conditions. An analysis of variance (ANOVA) was calculated (SuperAnova software for Apple Macintosh) to test the effects of the between subjects factor GROUP (symptomatic $v$ asymptomatic patients), and the within subjects factors PRES CONDITION (systolic negative pressure $v$ diastolic negative pressure) and HEART CYCLE (presentation of the electrical stimuli during systole $v$ diastole), with the sensory detection thresholds as the dependent variable. The effects of the same three factors were assessed on the estimates of the pain stimuli. Post hoc pairwise comparisons were performed using the Scheffé method.

\section{Results}

Effect of baroreceptor manipulation on heart rate

The two PRES conditions had significantly different effects on heart rate responses $[F(1,17)=47.6 ; \mathrm{p} \ll 0.001]$. As shown in table II, the tonic stimulation condition (negative cuff pressure during systole and positive cuff pressure during diastole) evoked larger heart rate decelerations than the control condition (in which cuff pressure was positive during systole and negative during diastole). Figure 1 presents the mean heart rate traces, averaged over symptomatic and asymptomatic patients separately in the stimulation and control conditions during the detection procedure. Asymptomatic patients did not differ from symptomatic patients with respect to the magnitude of heart rate deceleration $(F=0.6, N S)$.

Tourniquet pain test

Table II also shows that asymptomatic patients had a significantly higher pain threshold than the symptomatic patients $[\mathrm{F}(1,15)=4.9 ; \mathrm{p}<0.05]$. The asymptomatic patients reported pain an average of $17.6 \mathrm{~s}$ later than the symptomatic patients.

\section{Detection thresholds}

The different baroreceptor manipulation conditions did not have a significant effect on detection thresholds in either of the groups $[\mathrm{F}(1,17)=0.001, \mathrm{NS}]$. As can be seen in table II, the groups did not differ in their detection thresholds $[F(1,17)=0.04, N S]$. Similar negative results were obtained when baseline adjusted data (the detection threshold measured during a cuff-free baseline condition was subtracted from the threshold measured during the different baroreceptor manipulation conditions) were analysed.

\section{Ratings of painful stimuli}

The interaction between the within subjects factors HEART CYCLE and PRES CONDITION was significant $[\mathrm{F}(1,17)=18.5$; $\mathrm{p}<0.001]$. Post hoc tests revealed that the condition in which pain stimuli were applied during phases of minimum baroreceptor activity (systolic suction with diastolic pain) and rated

Table II Effects of PRES conditions on pain thresholds and heart rate responses. Values are means( $S D)$.

\begin{tabular}{|c|c|c|}
\hline & $\begin{array}{l}\text { Symptomatic } \\
\text { patients }\end{array}$ & $\begin{array}{l}\text { Asymptomatic } \\
\text { patients }\end{array}$ \\
\hline Tourniquet pain threshold & $39.444(18.105)$ & $57.000(14.071)$ \\
\hline Pain rating cond $1^{\mathrm{a}}$ & $0.544(0.170)$ & $0.637(0.130)$ \\
\hline Pain rating cond $2^{\mathrm{b}}$ & $0.610(0.170)$ & $0.704(0.112)$ \\
\hline Detection threshold cond $1^{\mathrm{a}}$ & $0.161(0.079)$ & $0.179(0.085)$ \\
\hline Detection threshold cond $2^{\mathrm{b}}$ & $0.161(0.067)$ & $0.159(0.044)$ \\
\hline Heart rate change during baro stimulation & $-2.267(0.899)$ & $-2.527(1.333)$ \\
\hline Heart rate change during baro stimulation & $-0.616(0.498)$ & $-0.906(0.747)$ \\
\hline
\end{tabular}

${ }^{2}$ Cond 1 refers to pain delivery during maximum baroreceptor activity (ie, pain during systole and negative cuff pressure).

Cond 2 refers to pain delivery during minimum baroreceptor activity (ie, pain during diastole and positive cuff pressure)
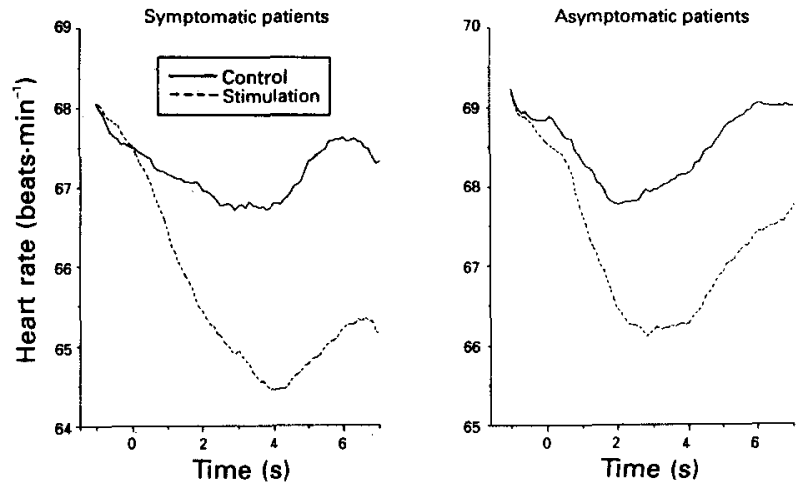

Figure 1 Mean heart rate traces during PRES baroreceptor manipulation and the threshold detection procedure. Stimulation (dotted line) and control condition (solid line) are plotted for the grand average of the $646 \mathrm{~s}$ trials.

significantly more painful than the condition with maximum baroreceptor activity (systolic suction with systolic pain, $\mathrm{F}=37.8, \ll 0.001$ ), or the two conditions with medium baroreceptor activity (diastolic suction with systolic pain, $\mathrm{F}=26.5, \mathrm{p} \ll 0.001$, or diastolic suction with diastolic pain, $\mathrm{F}=25.9, \mathrm{p} \ll 0.001)$. None of the other post hoc comparisons were significant. The interaction of GROUP, PRES CONDITION, and HEART CYCLE failed to reach significance $(\mathrm{F}<0.8$, see table II for values). It should be noted that an $\mathrm{F}$ value below unity would not be statistically significant at any sample size.

Figure 2 summarises the results of baroreceptor manipulation on pain ratings. Mean ratings averaged over patients are shown.

\section{Discussion}

Differential heart rate responses to the cuff pressure changes verified successful baroreceptor manipulation. This was comparable to earlier studies of normal subjects with the PRES technique. ${ }^{14}{ }^{16}$ Future research might control for respiration, as it has been shown ${ }^{21}$ that heart rate response to carotid baroreceptor stimulation is reduced during inspiration.

The results of the pain tests performed here in conjunction with external carotid baroreceptor manipulation revealed a significant effect on the ratings of painful electrical stimuli. In the condition evoking the greatest baroreceptor activity, in which negative cuff pressure was produced during systole and the electrical stimulus was timed to coincide with the maximum pressure elevation in the internal carotid artery (also

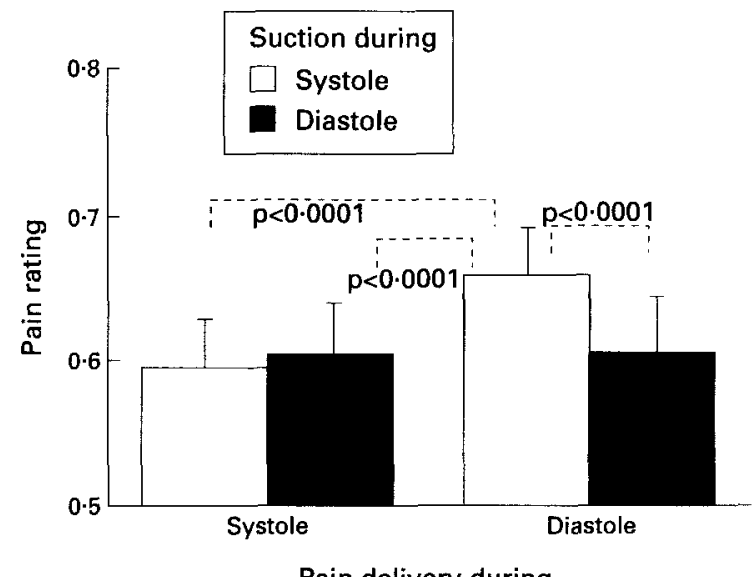

Pain delivery during

Figure 2 Results of baroreceptor manipulation and timing of pain delivery in the heart cycle on pain ratings. Mean ratings with standard error bars averaged over patients are shown. 
during systole), stimuli were judged as less painful than under conditions that dampened carotid stretch. The PRES conditions affording intermediate levels of baroreceptor activity (depicted in grey in fig 2) did result in intermediate pain ratings, but were not statistically differentiable from the contrasted extreme conditions. Earlier studies found differences in the dental pulp ${ }^{6}$ and transcutaneous electrical pain thresholds ${ }^{3}$ in these two patient groups. In the present study, we were interested in intrasubject variability as a function of baroreceptor related antinociception. For this reason, the objective (physical) stimulus intensity of the pain eliciting stimuli was individually adjusted to five times the intensity of the previously measured detection threshold. This individual adjustment procedure does not permit between subject comparisons.

The present results confirm earlier findings of baroreceptor activity dampening pain, but not detection. ${ }^{22}$ These findings provide further support for the hypothesis that the relief afforded by the increased baroreceptor activity during phasic increases in blood pressure may eventually lead to learned hypertension. ${ }^{23}$

Silent myocardial ischaemic patients had a higher tourniquet pain test threshold than symptomatic patients, consistent with earlier findings. ${ }^{3}{ }^{5}$ This resembles the process underlying the patient's decision to report the presence of angina pectoris pain in exercise tests. One clinically relevant implication is that patients' risk for asymptomatic (as opposed to symptomatic) myocardial ischaemia might be assessed with the tourniquet technique.

No heart rate response differences between patients with symptomatic and asymptomatic myocardial ischaemia were found, indicating comparable baroreflexes in the two groups.

Baroreceptor manipulation had no significant effect on sensory detection thresholds. The effect of baroreceptor manipulation was only found for painful stimuli; no such effect was found for sensory detection threshold. This finding suggests that the central nervous effects of baroreceptor manipulation act at a level beyond the primary sensory encoding stage of potentially painful stimuli. Baroreceptor manipulation does not attenuate sensation, it has a specific antinociceptive effect.

The groups did not differ in their antinociceptive response to the baroreceptor manipulations. Thus it could be concluded that the baroreceptors do not seem to play an important role in the clinical pain differences characterising these groups. The consistent positive results obtained with the PRES method of baroreceptor manipulation ${ }^{24}$ for at least heart rate changes, and often other variables, could not be achieved if intrasubject variability of response to PRES were high.

Future studies might involve controlling for respiration, medication, and degree of vessel disease. The heterogeneity of the latter two factors in the present study may have attenuated the ability to detect baroreceptor effects on differentiating symptomatic and silent myocardial ischaemia.

This research was supported by the German Research Society (Forschergruppe Schmerz: Bi 195/24-1).

Key terms: baroreceptors; pain; asymptomatic myocardial ischaemia.

Received 6 September 1993; accepted 15 November 1993. Time for primary review 21 days.

1 Cohn PF. Silent myocardial ischemia in patients with a defective anginal warning system. Am J Cardiol 1980:45:697-702

2 Erikssen J, Cohn PF, Thaulow E, Mowinckel P. Silent myocardial ischemia in middle-aged men: long-term clinical course. In: von Arnim T, Maseri A, eds. Silent ischemia. current concepts and management. Darmstadt: Steinkopff, 1987:45--51.
3 Droste C, Roskamm H. Experimental pain measurement in patients with asymptomatic myocardial ischemia. J Am Coll Cardiol 1983:1:940-5.

4 Droste $\mathrm{C}$, Roskamm H. Pain perception and endogenous pain modulation in angina pectoris. In: Kellerman JJ, Braunwald E, eds. Silent mvocardial ischemia: a critical appraisal. Basel: Karger, 1990: i 42-64.

5 Droste C, Greenlee MW, Roskamm H. A defective angina pectoris pain warning system: experimental findings of ischemic and electrical pain tests. Pain 1986;26:199-206.

6 Falcone C, Sconocchia R, Guasti L, Corsico G, Montemartini C, Specchia G. Dental pain threshold and angina pectoris in patients with coronary artery disease. J Am Coll Cardiol 1988; $12: 348-52$.

7 Rau H. Brody S, Larbig W, et al. The effects of PRES baroreceptor stimulation on thernal and mechanical pain threshold in borderline hypertensives and normotensives. Psichophysiologv (in press).

8 Dworkin B, Filewich RJ, Miller NE, Craigmyle N, Pickering TG. Baroreceptor activation reduces reactivity to noxious stimulation: Implications for hypertension. Science 1979;205:1299-301.

9 Randich A. Maixner W. Interactions between cardiovascular and pain regulatory systems. Neurosci Biobehav Rev 1984;8:343-67.

10 Zamir N, Segal M. Hypertension-induced analgesia: changes in pain sensitivity in experimental hypertensive rats. Brain Res 1979:160: 170-3

11 Zamir N, Shuber E. Altered pain perception in hypertensive humans. Brain Res 1980;201:471-4.

12 Elbert T, Lutzenberger W, Rockstroh B, Kessler M, Pietrowsky R, Birbaumer N. Baroreceptor stimulation increases pain threshold in borderline hypertensives. Psychophysiology 1988;25:25-9.

13 Furedy J, Rau H, Roberts L. Physiological and psychological differentiation of bidirectional baroreceptor carotid manipulation in humans. Physiol Behav 1992;52:953-8.

14 Rau H, Elbert T, Geiger B, Lutzenberger W. PRES: the controlled noninvasive stimulation of the carotid baroreceptors in humans. Psychophysiology 1992;29:165-72.

15 Lieberman HR, Pentland AP. Microcomputer-based estimation of psychophysical thresholds: the best PEST. Behavior Research. Methods Instrumentation 1982;14:21-5.

16 Rau H, Brody S, Droste C, Kardos A. Blood pressure changes validate phase related external suction, a controlled method for stimulation of human baroreceptors. Eur $J$ App Physiol 1993:67:26-9.

17 Bromm B. Modern techniques to measure pain in healthy man. Methods Find Exp Clin Pharmacol 1985;7:161-9.

18 Smith GM, Egbert LD, Markowitz RA, Mosteller F, Beecher HK. An experimental pain method sensitive to morphine in man: the submaximum effort tourniquet technique. $J$ Pharmacol Exp Ther 1966;154:324-32.

19 Pertovaara A. Kemppainen P, Vuolteenaho Ö, Leppäluoto J. The effect of tourniquet-induced ischemic pain on electrotactile thresholds, blood pressure and $\beta$-endorphin level in plasma. In: Bromm B, ed. Pain measurements in man: neurophysiological correlates of pain. Amsterdam: Elsevier, 1984:475-81.

20 Droste C, Greenlee MW. Two separate components of pain produced by submaximal effort tourniquet technique. Pain 1985;23:95-6.

21 Mancia G, Mark AL. Arterial baroreflexes in humans. In: Shepherd JT. Abboud FM, eds. Handbook of physiology. Section 2: The cardiovascular system, Vol III. Bethesda, Md. American Physiological Society, 1983:755-93.

22 Droste C, Kardos A, Brody S, Greenlee M, Roskamm H. Rau H. Baroreceptor stimulation: pain perception and sensory thresholds. Biol Psychol (in press).

23 Dworkin B. The baroreceptor reinforcement instrumental learning (BR-IL) model of essential hypertension: biological data quantitative mechanisms, and computer modeling. In: Shapiro A Baum U, eds. Behavioral aspects of cardiovascular disease. Hillsdale NJ: Lawrence Erlabaum, 1991:213-45.

24 Rau H, Brody S, Brunia C, Damen E, Elbert T. Activation of carotid baroreceptors inhibits spinal reflexes in man. Electroencephalogr Clin Neurophysiol 1993;89:328-34.

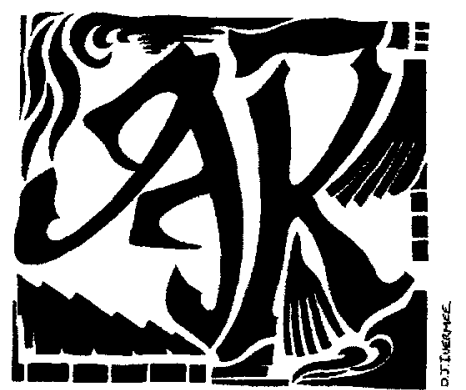

